\title{
MISE AU POINT D'UNE MÉTHODE D'ISOLEMENT DES TALITRES (CRUSTACÉS AMPHIPODES) À PARTIR DES LAISSES DE PLAGE
}

\author{
par \\ GÉRARD CIAVATTI \\ Université Antilles-Guyane, Laboratoire de Biologie Animale, \\ B.P. 592, Pointe à Pitre Cedex, Guadeloupe (Antilles)
}

\begin{abstract}
RÉSUMÉ
Les Talitridés (Orchestia platensis Kröyer, 1845) fuient les laisses de plage qui ont été inondées. La méthode d'isolement décrite exploite ce comportement. Elle permet d'isoler $97 \%$ des Talitres contenus dans les laisses. Elle pourrait être utilisée dans des études de biomasse et de productivité des Talitridés des laisses de plage.
\end{abstract}

\section{SUMMARY}

Talitridae (Orchestia platensis Kröyer, 1845) leave flooded jetsam. The method described here uses this behaviour and enables the extraction of $97 \%$ of beachhoppers living in beach wrack. It could be useful in studies on biomass and productivity of Talitridae of beach wrack.

\section{INTRODUCTION}

Dans le cadre d'une étude des Talitres des laisses de plage, nous nous sommes trouvés confrontés au problème de l'isolement des animaux de leur substrat, ici des débris végétaux et du sable.

La méthode la plus simple est l'isolement manuel à la pince. Divers auteurs travaillant sur les Amphipodes ont utilisé cette méthode (Louis, 1980; Sagar, 1980; Stoner, 1980; Waters, 1980). Le travail est rendu plus aisé grâce à une coloration préalable des animaux par le rose bengale. Williams (1974) a fait une revue des techniques de colorations facilitant l'isolement manuel. Mais cette méthode est longue et fastidieuse, c'est pourquoi divers auteurs ont tenté de résoudre le problème par d'autres moyens.

Il existe souvent une différence de densité entre animaux et substrat qui peut être utilisée pour l'isolement. C'est sur ce principe que repose la méthode de flottation ou de décanta- tion. Les animaux sont mis en suspension par un jet d'eau et récupérés sur un tamis (table de triage de Picard, 1965; méthode de Bodin, 1964, pour le meiobenthos). La différence de densité peut être accentuée par l'adjonction de substances dissoutes (sucre, sulfate de zinc ou de magnésium). Divers appareils d'isolement semi-automatiques utilisent cette méthode, tel celui de Thomassin modifié par Falconetti, 1973 (d'après Thomassin, 1978). Waters (1980) fait remarquer que la méthode de flottation est inutilisable lorsque les animaux sont mêlés à des débris végétaux, les densités étant voisines. Morino (1978) l'utilise cependant pour isoler les Talitres des laisses de mer mais il est obligé d'examiner attentivement les laisses et le sable.

D'autres méthodes, enfin, reposent sur le comportement animal lui-même. L'appareil de Bonet (1929) exploite le comportement lucifuge et hygrophile des microarthropodes du sol. Delamare-Debouteville, 1946 (d'après Seguy, 1949), récolte la faune du sol en inondant la terre. Notre méthode repose sur l'observation d'un comportement analogue chez les Talitres: ils fuient les laisses qui sont inondées.

\section{MÉTHODE}

Les prélèvements effectués sur la plage sont constitués par 15.000 à $20.000 \mathrm{~cm}^{3}$ d'épaves de végétaux morts (Thalassia testudinum et Syringodium filiforme) et de sable et contiennent les Talitridés (Orchestia platensis Kröyer, 1845). Ces prélèvements sont stockés dans un récipient de 47 $\mathrm{cm}$ de hauteur.

Les laisses contenant les Talitres sont réparties dans des bacs $A$ de $7,5 \mathrm{~cm}$ de hauteur et de 
$2.000 \mathrm{~cm}^{3}$ de volume. Un tube de plastique est enfoncé au centre des laisses (fig. 1). Chaque bac $A$ est ensuite déposé au fond d'un grand récipient $B$ de $47 \mathrm{~cm}$ de hauteur (fig. 2). Grâce au tube de plastique central, on verse de l'eau douce dans le bac A jusqu'à le remplir à moitié. Les Talitres fuient la moitié inférieure du bac. Au bout de 30 minutes, le remplissage du bac $\mathrm{A}$ est achevé avec une solution de formol à $0,4 \%$. Les Talitres sautent du bac $A$ et tombent dans le récipient $B$. Après $12 \mathrm{~h}$ les animaux qui se trouvent sur le fond du récipient $B$ sont tués avec une solution de formol à $4 \%$ et récupérés sur un tamis (maille de $20 \mu \mathrm{m}$ ).

\section{RÉSULTATS}

Nous avons compté le nombre d'animaux présents au fond du récipient $B$ (isolat) et le nombre d'animaux restés dans le bac A (résidu) au bout de $12 \mathrm{~h}$. Puis nous avons calculé le rendement de la méthode (isolat / isolat + résidu). Les résultats sont consignés dans le tableau I.

\section{Tableau I}

Résultats de 7 expériences d'isolement. Le rendement moyen est $96,97(\sigma=2,36)$.

\begin{tabular}{lrrr}
\hline Expériences & Isolat & $\begin{array}{c}\text { Isolat }+ \\
\text { Résidu }\end{array}$ & $\begin{array}{c}\text { Rendement } \\
\text { en \% }\end{array}$ \\
\hline No 1 & 189 & 197 & 95,94 \\
No 2 & 541 & 553 & 97,83 \\
No 3 & 988 & 1004 & 98,41 \\
No 4 & 426 & 465 & 91,61 \\
No 5 & 271 & 276 & 98,19 \\
No 6 & 299 & 306 & 97,71 \\
No 7 & 213 & 215 & 99,07 \\
Total & 2927 & 3016 & \\
\hline
\end{tabular}

\section{DISCUSSION ET CONCLUSION}

Le rendement fluctue peu autour de la moyenne et il est comparable à celui de la méthode de Bodin (1964). Des expériences préliminaires nous ont cependant montré qu'il est influencé par la hauteur du bac A: des bacs de

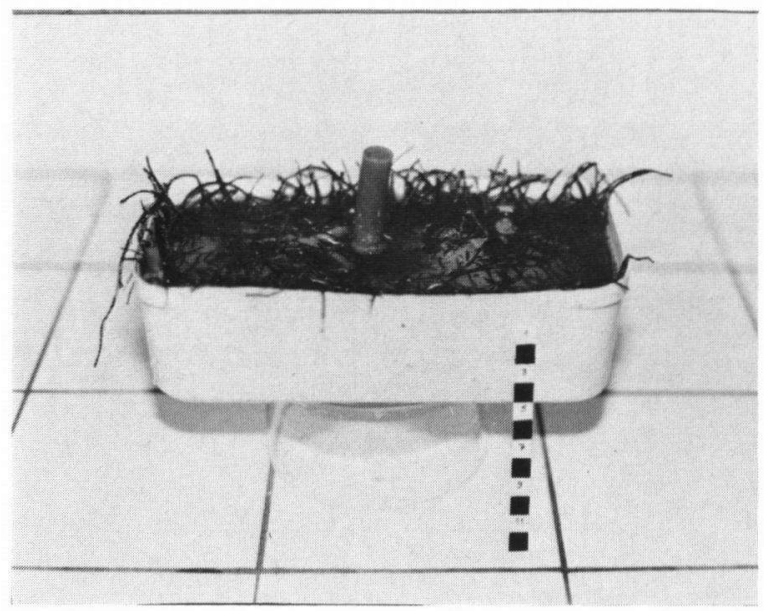

Fig. 1. Bac A hors du récipient B. Le bac contient les laisses, au centre le tube de plastique. Eau douce et eau formolée ont été ajoutées (échelle en $\mathrm{cm}$ ).

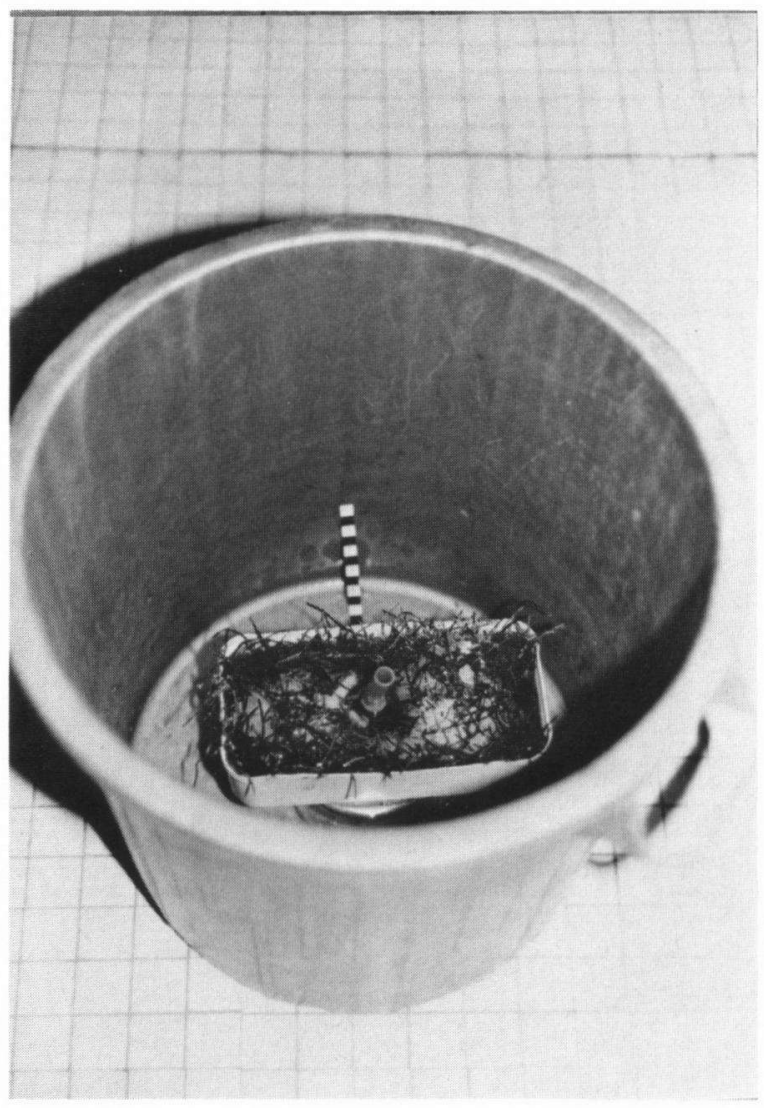

Fig. 2. Bac $A$ au fond du récipient B. Le couvercle du récipient $B$ a été enlevé (échelle en $\mathrm{cm}$ ). 


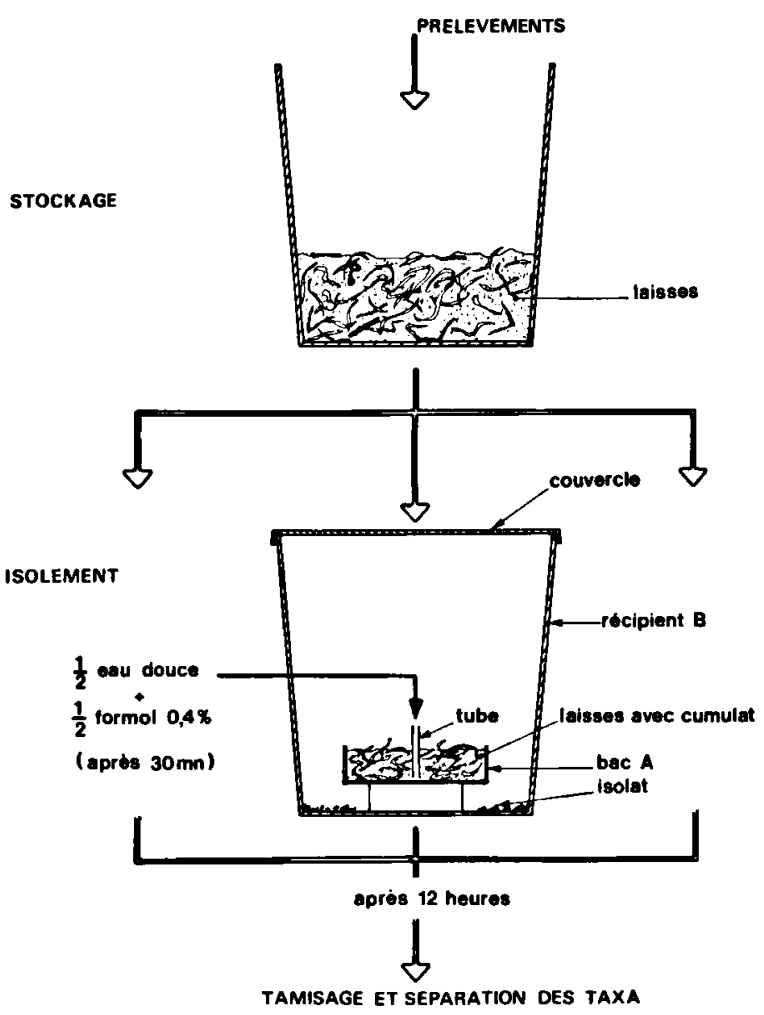

Fig. 3. Résumé des opérations d'isolement.

$30 \mathrm{~cm}$ de hauteur ont un rendement de $77 \%$. Un remplissage insuffisant ou incorrect du bac A diminue le rendement qui chute au-dessous de $90 \%$. Un bac à rendement faible se signale par un nombre élevé d'animaux morts à la surface des laisses. Lorsque le rendement est supérieur à $90 \%$, ce nombre représente 0 à $1 \%$ de l'isolat, lorsque le rendement chute au-dessous de $90 \%$, il représente alors 5 à $18 \%$ (fig. 4).

Ces remarques étant faites, un rendement moyen de $97 \%$ nous semble suffisant pour une étude de la biomasse des laisses des plages. Ce rendement est même légèrement supérieur à celui que l'on peut obtenir par un tri manuel simple à la pince après coloration au rose bengale.

Les autres Arthropodes des laisses (Isopodes, larves d'Insectes, Dermaptères) fuient également l'inondation du bac $\mathrm{A}$ et sont récupérés avec les Talitres. Leur faible abondance ne permet pas de donner un rendement. Notons qu'aucun des cumulats des expériences d'isole- ment ne contenait d'Arthropodes autres que les Talitres.

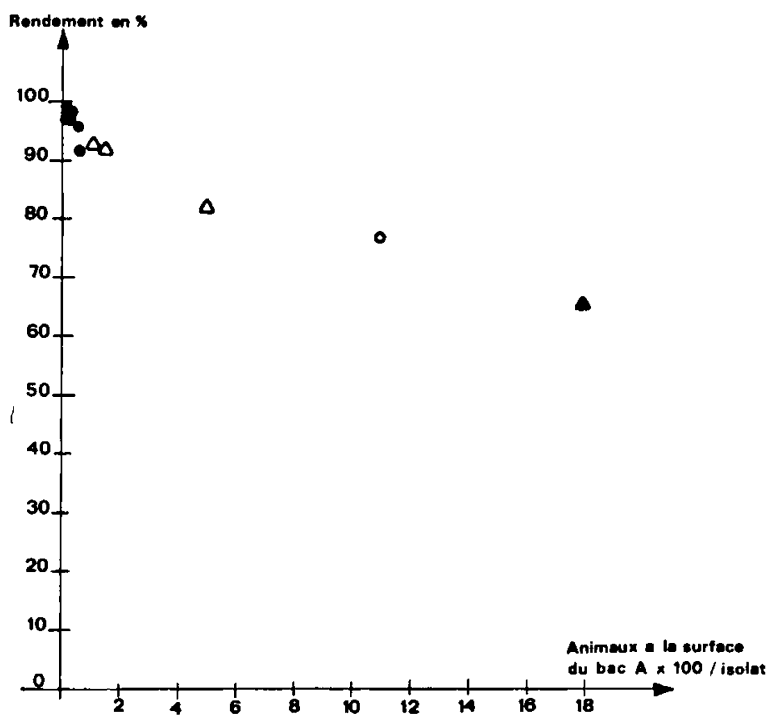

Fig. 4. Rendement de la méthode en fonction du nombre d'animaux restés à la surface du bac $\mathrm{A}$ après 12 heures.

- Eau douce + eau formolée.

$\triangle$ Eau douce seulement.

- Bac de $30 \mathrm{~cm}$ de hauteur.

- Adjonction trop rapide d'eau formolée dans le bac $\mathrm{A}$.

\section{BIBLIOGRAPHIE}

Bodin, P., 1964. Recherches sur la systématique et la distribution des Copépodes Harpacticoïdes des substrats meubles des environs de Marseille. Recl. Trav. Stn. mar. Endoume, 35 (51): 107-183.

BONET, F., 1929. Un nuevo aparato para la recoleccion de microartropodos. Confcias. Reseñ. cient. R. Soc. esp. Hist. nat., 4: 91-94.

Falconetri, C., 1973. Fonctionnement d'un appareil de tri semi-automatique. Rapp. P.-v. Réun. Commn. int. Explor. scient. Mer Méditerr., 22 (4): 125-127.

Lours, M., 1980. Étude d'un peuplement mixte d'Orchestia montagui Audouin et d'O. dehayesei Audouin, dans la baie de Bou Ismail (Algérie). Bull. Ecol., 11 (2): 97-111.

Morino, H., 1978. Studies on the Talitridae (Amphipoda, Crustacea) in Japan. III. Life history and breeding activity of Orchestia platensis Kröyer. Publs. Seto mar. biol. Lab., 24 (416): 245-267.

Picard, J., 1965. Recherches qualitatives sur les biocénoses marines des substrats meubles dragables de la région marseillaise: 1-166 (Thèse d'État, Faculté d'Aix-Marseille).

SAgAR, P. M., 1980. Life cycle and growth of the Antarctic gammarid amphipod Paramoera walkeri (Stebbing, 1906). J. Roy. Soc. New Zealand, 10 (3): 259-270. 
SEGUY, E.-A., 1949. Le microscope, emploi et applications, vol. II. In: Encyclopédie pratique du naturaliste, 34: 322-1062 (P. Lechevalier, Paris).

Stoner, A. W., 1980. Abundance, reproductive seasonality and habitat preferences of amphipod crustaceans in seagrass meadows of Apalachee Bay, Florida. Contr. mar. Sci., 23: 63-77.

Thomassin, B., 1978. Soft-bottom communities. In: D. R. Stoddart \& R. E. Johannes eds., Coral reefs: research methods: 263-298 (Unesco, Paris).
WATERS, T. F., 1980. Annual production and drift of the stream amphipod Gammarus pseudolimnaeus in Valley Creek, Minnesota. Limnol. Oceanogr., 25 (4): 700-710.

Williams, G. E., 1974. New technique to facilitate handpicking macrobenthos. Trans. Am. microsc. Soc., 93 (2): 220-226.

Reçu le 1 février 1983 